\title{
PERFIL BACTERIANO E FENOTÍPICO DE SENSIBILIDADE AOS ANTIMICROBIANOS EM ISOLADOS DE HEMOCULTURA EM UM HOSPITAL PÚBLICO DE GRANDE PORTE DO MUNICÍPIO DO RIO DE JANEIRO: RELATO DE EXPERIÊNCIA
}

\author{
Maurício Cavalcanti da Silva \\ Universidade Federal do Rio de Janeiro \\ mauriciocavalcantienf@gmail.com
}

Thais de Rezende Bessa Guerra

Instituto de Nutrição do Cérebro e do Coração

tcrezende34@gmail.com

Aline Trevizan da Silva Mendes

Universidade Federal do Rio de Janeiro

linetrevizan@gmail.com

\section{Cassiano Felippe Gonçalves de Albuquerque}

Universidade Federal do Estado do Rio de Janeiro

cassianofg@gmail.com

\author{
Adriana Ribeiro da Silva \\ Fundação Oswaldo Cruz \\ arsilva71@gmail.com
}

\section{Resumo}

Objetivo: o presente artigo buscou relatar a experiência sobre a rotina do setor de análises clínicas com foco no perfil bacteriano e fenotípico de sensibilidade aos antimicrobianos em microorganismos isolados por meio de hemocultura. Métodos: estudo transversal, com caráter exploratório-descritivo, desenvolvido como projeto de extensão durante 0 período de estágio. O protocolo do estudo foi relatar a experiência sobre a rotina do setor de análises clínicas para conhecer os testes microbiológicos de isolados de hemocultura e de seus respectivos testes de susceptibilidade aos antimicrobianos, realizados no período entre 01 de junho à 31 de setembro de 2018. Resultados: foram observadas um total de 357 análises de hemoculturas, com crescimento de microorganismos com prevalência de bactérias da espécie Staphylococcus aureus e Staphylococcus epidermidis $\mathrm{n}=119$. As 10 bactérias mais identificadas, entre elas gram positivas e 
gram negativas, apresentaram restrições aos antimicrobianos a serem utilizados como terapêutica. Conclusões: conclui-se que é necessária adoção de estratégias que minimizem a incidência de infecções associadas à corrente sanguínea, com enfoque na educação continuada de profissionais de saúde, além da implementação de medidas de prevenção, detecção e tratamento das complicações advindas da resistência microbiana, como a sepse, consequência final de infecções.

Palavras-chave: Bactérias. Hemocultura. Teste de Sensibilidade Microbiana.

\title{
BACTERIAL AND PHENOTYPICAL PROFILE OF SENSITIVITY TO ANTIMICROBIALS IN HEMOCULTURE ISOLATES IN A LARGE PUBLIC HOSPITAL IN THE MUNICIPALITY OF RIO DE JANEIRO: EXPERIENCE REPORT
}

\begin{abstract}
Objective: the present article sought to report the experience about the routine of the clinical analysis sector with a focus on the bacterial and phenotypic profile of sensitivity to antimicrobials in microorganisms isolated through blood culture. Methods: cross-sectional study, with an exploratorydescriptive character, developed as an extension project during the

probation. The protocol of the study was to report the experience about the routine of the clinical analysis sector in order to know the microbiological tests of blood culture isolates and their respective susceptibility tests to antimicrobials, carried out in the period from June 1st to September 31st, 2018. Results: a total of 357 blood culture analyzes were observed, with the growth of microorganisms with a prevalence of bacteria of the species Staphylococcus aureus and Staphylococcus epidermidis $n=119$. The 10 most identified bacteria, including gram positive and gram negative, presented restrictions on the antimicrobials to be used as therapy. Conclusions: it is concluded that it is necessary to adopt strategies that minimize the incidence of infections associated with the bloodstream, with a focus on continuing education of health professionals, in addition to the implementation of preventive measures, detection and treatment of complications arising from microbial resistance, like sepsis, the final consequence of infections.
\end{abstract}

Keywords: Bacteria. Blood culture. Microbial Sensitivity Tests.

\section{PERFIL BACTERIAL Y FENOTÍPICO DE SENSIBILIDAD A LOS ANTIMICROBIANOS EN AISLADOS DE HEMOCULTURA EN UN GRAN HOSPITAL PÚBLICO EN EL MUNICIPIO DE RÍO DE JANEIRO: INFORME DE EXPERIENCIA}

\section{Resumen:}

Objetivo: el presente artículo buscó informar la experiencia sobre la rutina del sector del análisis clínico con un enfoque en el perfil bacteriano y fenotípico de la sensibilidad a los antimicrobianos en microorganismos aislados a través de hemocultivos. Métodos: estudio transversal, de carácter exploratorio-descriptivo, desarrollado como proyecto de extensión durante el período de prácticas. El protocolo del estudio fue informar la experiencia sobre la rutina del sector del análisis clínico para conocer las pruebas microbiológicas de los aislados de hemocultivos y sus respectivas pruebas de susceptibilidad a los antimicrobianos, realizadas en el período del 1 de junio al 31 de septiembre de 2018. Resultados: se observaron un total de 357 análisis de hemocultivos, con el crecimiento de microorganismos con una prevalencia de bacterias de las especies Staphylococcus aureus y Staphylococcus epidermidis $n=119$. Las 10 bacterias más identificadas, incluidas las gram positivas y las gram negativas, presentaron restricciones para que los antimicrobianos fueran utilizado como terapia Conclusiones: se concluye que es necesario adoptar estrategias que minimicen la incidencia de infecciones asociadas al torrente sanguíneo, con un enfoque en la educación continua de los profesionales de la salud, además de la implementación de medidas preventivas, detección y tratamiento de complicaciones derivadas de la resistencia microbiana. como la sepsis, la consecuencia final de las infecciones. 
Palabras clave: Bacterias. Cultura de sangre. Prueba de sensibilidad microbiana. 


\section{INTRODUÇÃO}

A hemocultura consiste no principal método para a detecção de microorganismos na corrente sanguínea. Patógenos interagem com o hospedeiro e induzem uma resposta local e/ou sistêmica, caracterizando uma infecção, quando há uma exacerbação e uma desregulação desta resposta com geração de risco de vida, surge assim, a sepse (SINGER, 2016).

Nesse contexto, o laboratório microbiológico possui suma importância, pois gerencia o recebimento e o processamento da amostra, visando o isolamento e identificação de patógenos para nortearem a terapia antimicrobiana. Dessa maneira, bactérias resistentes e multirresistentes se apresentam como problema de saúde pública mundial, reforçando a adoção de medidas básicas na prevenção e no controle de infecções nosocomiais e a racionalização do uso de antimicrobianos, pois inúmeras e onerosas são as consequências da habilidade de adaptação das bactérias.

Diante disso, conhecer as peculiaridades que antecedem o culminar de agravos que comprometem o estado de saúde de um indivíduo é de grande valia, pois estimula e subsidia a tomada de decisões na prática clínica.

O presente estudo tem como objetivo relatar a experiência sobre a rotina do setor de análises clínicas com foco no perfil bacteriano e fenotípico de sensibilidade aos antimicrobianos em microorganismos isolados por meio de hemocultura.

\section{METODOLOGIA}

Trata-se de um estudo transversal, com caráter exploratório-descritivo, desenvolvido como projeto de extensão durante o período de estágio. O protocolo do estudo foi relatar a experiência sobre a rotina do setor de análises clínicas para conhecer os testes microbiológicos de isolados de hemocultura e de seus respectivos testes de susceptibilidade aos antimicrobianos, realizados no período entre 01 de junho à 31 de setembro de 2018.

O projeto de extensão foi criado para que os estudantes de enfermagem pudessem observar a rotina do setor de análises clínicas e assim, conhecer mais de perto os testes microbiológicos de isolados de hemocultura e de seus respectivos testes de susceptibilidade aos antimicrobianos, em hospital público geral de grande porte do município do Rio Janeiro; este hospital congrega o maior serviço de urgência e emergência da América Latina. 
A identificação do agente etiológico bacteriano presente na hemocultura e do respectivo teste de susceptibilidade aos antimicrobianos armazenados no software Observa ${ }^{\circledR}$, tornando este um banco de dados. A determinação de susceptibilidade aos antimicrobianos se dá em concordância com os critérios do Clinical Laboratory Standart Institute (CLSI). Os dados foram coletados e analisados entre os meses de setembro e outubro de 2018, e em seguida organizados no software Microsoft Office Excel®, para geração dos gráficos.

Em concordância com a Resolução n ${ }^{\circ}$ 466, de 12 de dezembro de 2012, do Conselho Nacional de Saúde; o presente estudo não necessitou de submissão ao Comitê de Ética em Pesquisa com Seres Humanos, pois se deu por meio da rotina de monitoramento de um serviço de Análises Clínicas do hospital, portanto, visando obter um conhecimento generalizável.

\section{RESULTADOS}

Nesse contexto, os estudantes observaram 357 hemoculturas, onde não foi possível isolar e identificar bactérias em $\mathrm{n}=238(66,7 \%)$, constituindo assim as hemoculturas com resultado negativo, $\mathrm{n}=119$ (33,3\%) houve crescimento bacteriano (Gráfico 1; figura A). Nota-se que, quando classificadas quanto à morfologia há o predomínio de $24 \%$ na identificação de bactérias pertencentes ao grupo das gram positivas quando comparada com as bactérias gram negativas em isolados de hemoculturas (Gráfico 1, figura B). 
Gráfico 1: Distribuição de Hemoculturas em um Hospital Público Geral de grande porte do Município do Rio de Janeiro, segundo padrão de crescimento bacteriano e morfologia - período 01/06 a 31/09/18.
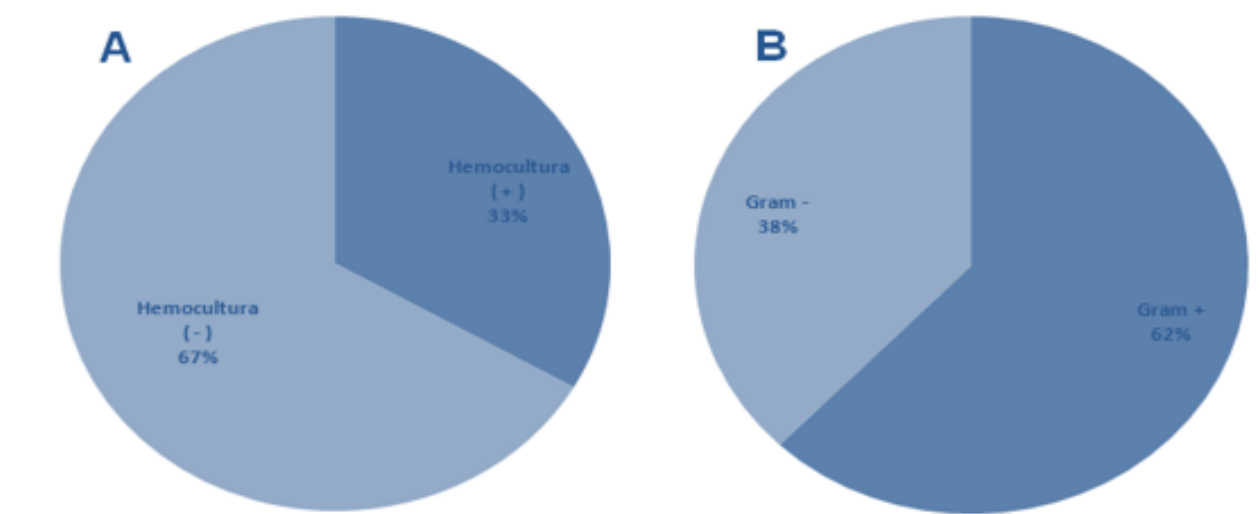

Legenda: Distribuição em frequência e percentual; (A): Presença e ausência de crescimento bacteriano;

(B): em relação a sua morfologia.

Totalizando 18 espécies bacterianas isoladas e identificadas em hemocultura, o gráfico 2 sumariza a distribuição das espécies bacterianas isoladas e identificadas nas hemoculturas positivas e determina alta frequência das pertencentes ao gênero Staphylococcus, com destaque para S. aureus (26\%) e S. epidermidis (18\%); seguido de Serratia marcescences (8\%), klebsiella pneumoniae (8\%), Proteus mirabilis (7\%), Staphylococcus haemolyticus (6\%), Staphylococcus lugdunensis (5\%), Pseudmonas aeruginosa (4\%), Escherichia coli (3\%) e Acinetobacter baumannii (3\%). As principais bactérias isoladas em hemocultura no hospital de estudo foram totalizadas em n=10 (Gráfico 2).

Observou-se que 11 unidades clínicas/setores de atendimento havia pedidos de solicitação de exame do tipo hemocultura ao longo do ano: Unidade de Terapia Intensiva 2/Unidade de Paciente Grave (UTI 2/UPG), Clínica Indeterminada (que surge mediante a incompletude no preenchimento de solicitação de exame), Hemodiálise, Clínica Médica, Unidade de Terapia Intensiva 3, Centro de Tratamento de Queimados (CTQ) Adulto, Unidade Coronariana, Nefrologia, Neurocirurgia, Sala de Reanimação e Pediatria. As unidades de atendimento com menos de quatro bactérias identificadas foram excluídas do gráfico 3, logo traduz-se as 5 clínicas/setores de atendimento com o maior quantitativo de espécies bacterianas isoladas e identificadas. 
Gráfico 2: Percentual de Microrganismos Isolados no Exame de Hemocultura em um Hospital Público Geral de grande porte do Município do Rio de Janeiro- período 01/06 a $31 / 09 / 18$.

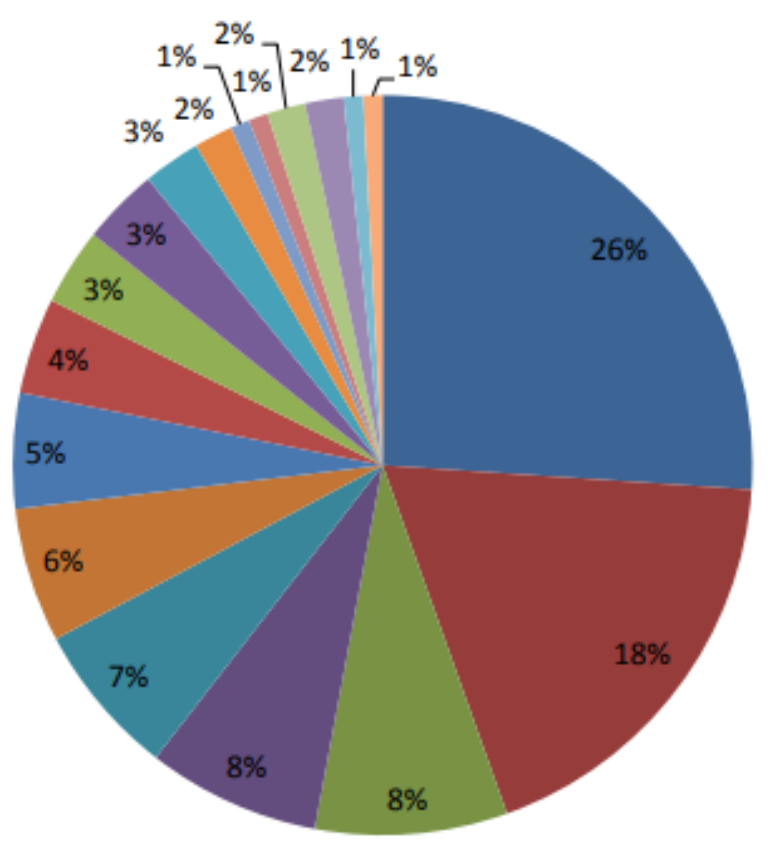

Staphylococcus aureus
$\square$ Staphylococcus epidermidis
Serratia marcescens
$\square$ klebsiella pneumoniae
$\square$ Proteus mirabilis
$\square$ Staphylococcus haemolyticus
$\square$ Staphylococcus lugdunensis
$\square$ Pseudomonas aeruginosa
$\square$ Escherichia coli
$\square$ Acinetobacter baumannii
$\square$ Enterococcus faecalis
Staphylococcus hominis
Morganella morganii morganii
Morganella morganii sibonii
Staphylococcus capitis
$\square$ Enterobacter aerogenes
Staphylococcus sciuri
klebsiella oxytoca

Fonte: Legenda: Distribuição em percentual (2018).

Quanto à variedade de espécies bacterianas identificadas pelos estudantes, a UTI 2/UPG obteve o maior quantitativo, 12 espécies bacterianas isoladas; a Clínica Indeterminada com 09 espécies bacterianas detectadas merece destaque, pois apesar de apresentar um segundo maior número de bactérias identificadas, concentra o maior número de solicitações de exame; seguida da unidade de Hemodiálise, Clínica Médica e UTI 3, respectivamente com 07, 04 e 03 solicitações de exame do tipo hemocultura.

As duas unidades clínicas com o maior número de espécies identificadas, observa-se uma tendência de $S$. aureus, como sendo o microorganismo mais isolado por números de hemocultura, de todas as clínicas em que houve solicitação de exame, este só não aparece em 4/11 unidades de atendimento (Clínica Médica, UTI 3, Nefrologia, Sala de Reanimação e Pediatria). Na clínica de Hemodiálise, este patógeno apresenta-se como a segunda bactéria mais identificada, ficando atrás de S. lugdunensis. 
Gráfico 3: Distribuição da Frequência de Hemoculturas Positivas por espécie bacteriana isolada, segundo à clínica/setor de atendimento em um Hospital Público de grande porte do município do Rio de Janeiro- período 01/06 a 31/09/18.

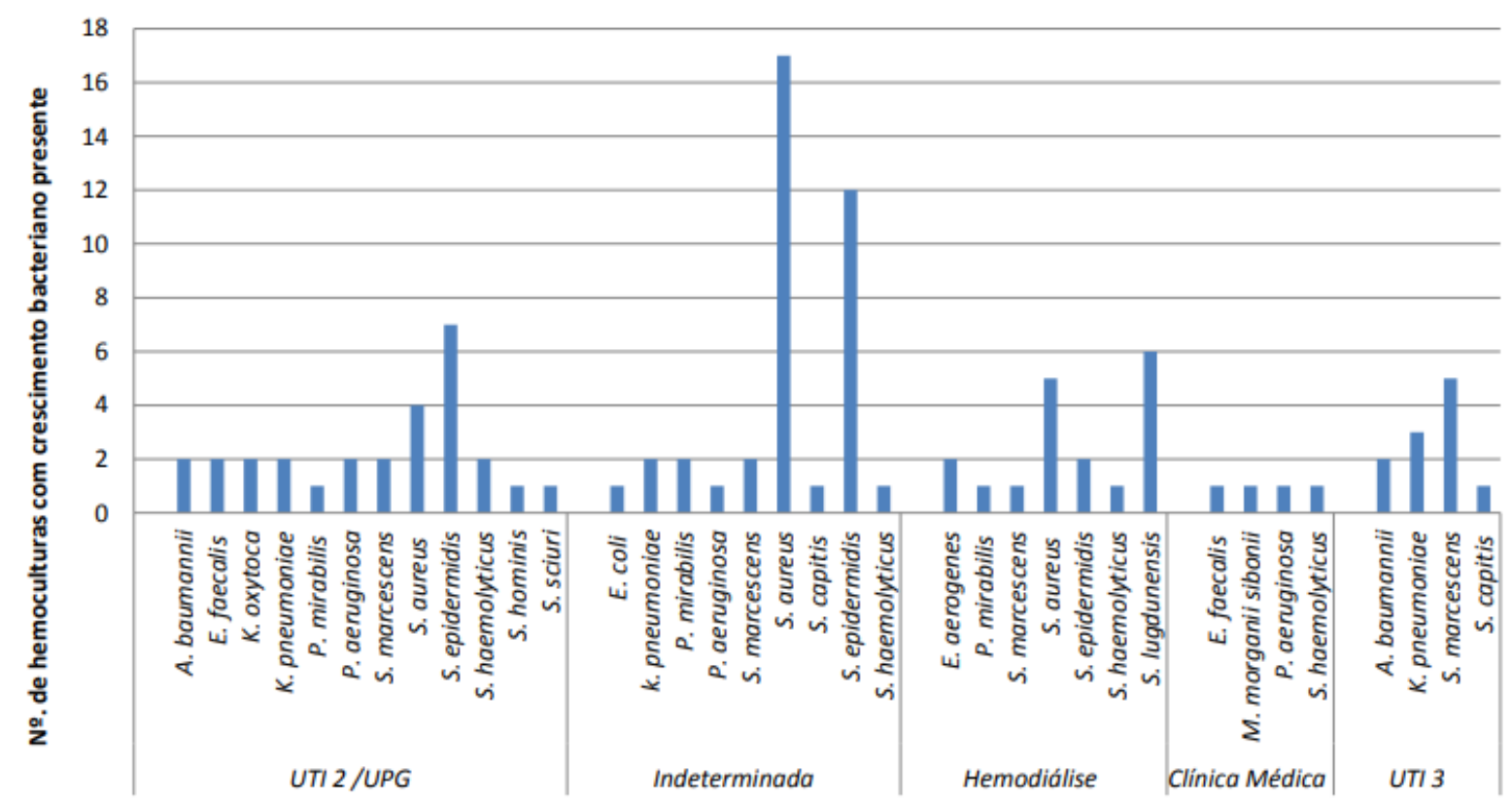

Fonte: Legenda: distribuição em frequência (2018)

Nos microorganismos mais identificados em hemocultura, destacamos a porcentagem em relação à sensibilidade obtida por meio do teste de susceptibilidade aos principais antimicrobianos utilizados na clínica, conforme demonstra a tabela 1 para bactérias gram positivas e a tabela 2 para as bactérias gram negativa.

$\mathrm{Na}$ tabela 1, observa-se que todos os microorganismos pertencentes ao grupo das bactérias gram positivas pertencem ao mesmo gênero bacteriano e denotam perfis diferentes de sensibilidade aos antimicrobianos, sendo $S$. lugdunensis o que apresenta uma maior faixa de sensibilidade aos antimicrobianos com resistência a Oxacilina e a Benzilpenicilina e de forma alarmante $S$. haemolyticus apresenta sensibilidade completa em apenas 05 antimicrobianos - Linezolid, Rifampicina, Vancomicina, Ácido Fusídico e Tigeciclina, juntamente com $S$. epidermidis que apresenta sensibilidade para os mesmos, acrescido de sensibilidade para Teicoplamina.

A tabela 2 retrata que A. baumannii é o patógeno gram negativo com menor sensibilidade aos antimicrobianos e $E$. coli o que apresenta maior número de antimicrobianos com sensibilidade completa. $P$. aeruginosa apresenta variações de sensibilidade em todas as 
gerações de cefalosporinas, inclusive sensibilidade parcial a terceira geração representada pelo antimicrobiano Ceftazidima, apresentando sensibilidade total para a cefalosporina de quarta geração (Cefepime) e a Colistina. K. pneumoniae apresenta sensibilidade variada em relação aos B-lactâmicos e as cefalosporinas, com sensibilidade total apenas para Amicacina, Colistina e Tigeciclina. De um modo geral, particularizamos uma restrição de antimicrobianos que possam ser utilizados como terapêutica em todos os microorganismos em ambas as tabelas. 
Tabela 1: Porcentagem e Perfil Fenotípico de Sensibilidade aos Antimicrobianos nas mais Frequentes Bactérias Gram Positivas Isoladas e Identificadas a partir de Hemocultura em um Hospital Público de grande porte do Município do Rio de Janeiro.

\begin{tabular}{|c|c|c|c|c|c|c|c|c|c|c|c|c|c|c|c|}
\hline $\begin{array}{l}\text { Bactéria gram } \\
\qquad(+)\end{array}$ & BZP & CPX & CLIN & ERIT & GEN & LNZ & Mox & NOR & OXA & RIF & TEI & SXT & VAN & FUS & TIG \\
\hline S. aureus & $0 \%$ & $53 \%$ & $47 \%$ & $24 \%$ & $100 \%$ & $100 \%$ & $53 \%$ & $53 \%$ & $18 \%$ & $100 \%$ & $100 \%$ & $88 \%$ & $100 \%$ & $100 \%$ & $* *$ \\
\hline S. epidermidis & $25 \%$ & $67 \%$ & $75 \%$ & $58 \%$ & $83 \%$ & $100 \%$ & $67 \%$ & ** & $50 \%$ & $92 \%$ & $100 \%$ & $0 \%$ & $100 \%$ & $100 \%$ & $100 \%$ \\
\hline S. haemolyticus & $0 \%$ & $0 \%$ & $0 \%$ & $0 \%$ & $0 \%$ & $100 \%$ & $0 \%$ & $0 \%$ & $0 \%$ & $100 \%$ & $0 \%$ & $0 \%$ & $100 \%$ & $100 \%$ & $100 \%$ \\
\hline S. lugdunensis & $0 \%$ & $100 \%$ & $100 \%$ & $100 \%$ & $100 \%$ & $100 \%$ & $100 \%$ & $100 \%$ & $13 \%$ & $100 \%$ & $100 \%$ & $100 \%$ & $100 \%$ & $100 \%$ & $100 \%$ \\
\hline
\end{tabular}

Fonte: Distribuição percentual; Antimicrobianos: (BZP): Benzilpenicilina; (CPX): Ciprofloxacina; (CLIN): Clindamicina; (ERIT): Eritromicina; (GEN):

Gentamicina; (LNZ): Linezolid; (MOX): Moxifloxacina; (NOR): Norfloxacina; (OXA): Oxacilina; (RIF): Rifampicina; (TEI): Teicoplamina; (SXT):

Trimetroprim + Sulfametoxazol; (VAN): Vancomicina; (FUS): Ácido Fusídico; (TIG): Tigeciclina; ** Antimicrobiano não testado.

Tabela 2: Porcentagem e Perfil Fenotípico de Sensibilidade aos Antimicrobianos nas mais Frequentes Bactérias Gram Negativas Isoladas e Identificadas a partir de Hemocultura em um Hospital Público de grande porte do Município do Rio de Janeiro.

\begin{tabular}{ccccccccccccccccccc} 
Bactéria gram (-) & AMC & AMP AMP + SBT & CFP & CFX & CFZ & CFO & CFM CFM + AXT & CPX & COL & ERT & GEN & IMI & MER PTB & TIG \\
\hline S. marcescens & $40 \%$ & $0 \%$ & $0 \%$ & $40 \%$ & $0 \%$ & $40 \%$ & $40 \%$ & $0 \%$ & $0 \%$ & $100 \%$ & $0 \%$ & $40 \%$ & $100 \%$ & $40 \%$ & $40 \%$ & $40 \%$ & $75 \%$ \\
\hline K. pneumoniae & $100 \%$ & $0 \%$ & $67 \%$ & $67 \%$ & $67 \%$ & $67 \%$ & $67 \%$ & $67 \%$ & $67 \%$ & $67 \%$ & $100 \%$ & $67 \%$ & $67 \%$ & $67 \%$ & $67 \%$ & $67 \%$ & $100 \%$ \\
\hline P. aeruginosa & $50 \%$ & $0 \%$ & $0 \%$ & $100 \%$ & $0 \%$ & $50 \%$ & $0 \%$ & $0 \%$ & $0 \%$ & $50 \%$ & $100 \%$ & $* *$ & $50 \%$ & $50 \%$ & $50 \%$ & $50 \%$ & $0 \%$ \\
\hline E. coli & $100 \%$ & $0 \%$ & $0 \%$ & $0 \%$ & $100 \%$ & $0 \%$ & $0 \%$ & $0 \%$ & $0 \%$ & $100 \%$ & $100 \%$ & $100 \%$ & $100 \%$ & $100 \%$ & $100 \%$ & $100 \%$ & $100 \%$ \\
\hline A. baumannii & $100 \%$ & $0 \%$ & $0 \%$ & $0 \%$ & $0 \%$ & $0 \%$ & $0 \%$ & $0 \%$ & $0 \%$ & $0 \%$ & $100 \%$ & $* *$ & $0 \%$ & $0 \%$ & $0 \%$ & $0 \%$ & $100 \%$
\end{tabular}

Fonte: Distribuição percentual; Antimicrobianos: (AMC): Amicacina; (AMP): Ampicilina; (AMP+SBT): Ampicilina + Sulbactam; (CFP): Cefepime; (CFX): Cefoxitina; (CFZ): Ceftazidima; (CFO): Ceftriaxona; (CFM): Cefuroxima; (CFM+AXT): Cefuroxima+Axetil; (CPX): Ciprofloxacina; (COL): Colistina; (ERT): Ertapenem; (GEN): Gentamicina; (IMI): Imipenem; (MER): Meropenem; (PTB): Piperacilina+Tazobactam; (TIG): Tigeciclina; **Antimicrobiano não testado.

Cidadania em Ação: Revista de Extensão e Cultura, Florianópolis (SC), v. 4, n. 1, jan./jun. 2020. 


\section{DISCUSSÃO}

De acordo com nosso levantamento bibliográfico, esse é o primeiro relato de experiência de estudantes que participaram de um projeto de extensão vinculado ao período de estágio, buscando conhecer o perfil bacteriano e fenotípico de sensibilidade aos antimicrobianos em microorganismos isolados por intermédio de hemoculturas em um hospital público de grande porte do município do Rio de Janeiro.

De acordo com a Pesquisa Nacional em Saúde, realizada em 2013 pelo Instituto Brasileiro de Geografia e Estatística (IBGE), das pessoas que ficaram internadas 24 horas ou mais nos últimos 12 meses anteriores à entrevista, 65.7\% (8,0 milhões) obtiveram atendimento, no nível terciário, por meio do Sistema Único de Saúde (SUS).Nesse sentido, a região sudeste apresentou uma proporção de $58.8 \%$ de pessoas, do total de entrevistados, que ficaram internadas em hospitais por 24 horas ou mais (BRASIL, 2014). Atrelado a isso, observa-se uma tendência ao decréscimo no número total de beneficiários de planos de saúde suplementar em assistência médica com ou sem odontologia (AGÊNCIA NACIONAL, 2015).

Nesse contexto, a hemocultura tem sua utilidade clínica quando coletada antes do início da terapia antimicrobiana ${ }^{1}$, entretanto, nem sempre são positivas, indicando uma bacteremia verdadeira e podem estar associadas a danos. Ademais, não devem ser coletadas de pacientes imunocomprometidos rotineiramente estáveis com celulite, pielonefrite simples, cistite e pneumonia adquirida na comunidade. Por outro lado, devem ser garantidas no paciente com sepse, pneumonia associada aos serviços em saúde e pielonefrite complicada (LONG; KOYFMAN, 2016).

Walraven and Wong ${ }^{5}$, concluíram que a hemocultura negativa não influenciou na morte hospitalar quando comparada aos pacientes que possuíam infecção de corrente sanguínea.Este desfecho depende da condição imunológica do paciente e do microorganismo causador, com destaque para o fungo do gênero Candida e bactérias do gênero Clostridium, sendo que esta bactéria não foi isolada. Nossas relatos corroboram com esses achados, quando observamos que as unidades de terapias intensivas são os principais serviços que demandam da solicitação de hemocultura. Staphylococcus coagulase negativa, Micrococcus, Clostridium sp., bacilos gram positivos, espécies de Neisseria não patogênicas, Streptococcus viridans, Streptococcus não hemolíticos e difteróides; são considerados contaminantes de hemocultivo (HERNÁNDEZ-BOU, et al., 2015), logo, há uma tendência na literatura em busca de técnicas mais sofisticadas para eliminação de contaminação nas hemoculturas e identificação de valores preditivos de contaminação. 
Já o resultado ao qual às solicitações de pedido de exame se encontravam em caráter incompleto, o Ministério da Saúde brasileiro lançou em 2014, a Política Nacional de Segurança do Paciente; com o objetivo de fortalecer essa prática, duas das seis metas estão diretamente relacionadas ao referido resultado observado: I. Identificar corretamente o paciente e II. Melhorar a comunicação entre os profissionais de saúde (BRASIL, 2014).

As principais bactérias conhecidas reforçam aquelas apontadas pela Organização Mundial de Saúde (OMS) como prioridade em pesquisa para desenvolvimento de novos antimicrobianos em decorrência da multirresistência .

Nossas observações corroboram com um estudo nacional realizado por um laboratório de análises clínicas que recebe amostras de 6 hospitais da rede privada, nestes se obteve grande prevalência de identificação de cocos gram positivos com destaque para $S$. aureus e $S$. epidermidis. A baumannii é indicado como o gram negativo com maior índice de resistência aos antimicrobianos, fato compartilhado também em nosso estudo. Entretanto, ainda não foi identificado no hospital de estudo a resistência a Colistina neste microorganismo, fato importante associado ao gene MCR-1 (do inglês: Mobile Colistin Resistance - 1) (BUTAYE; LAMMENS; GOOSSENS; DAS; XAVIER; MALHOTRAKUMAR, 2016) que vem sendo reportado em diversos microorganismos (ERFANIMANESH; HASHEMI, 2016). Em nosso estudo somente $S$. marcescens apresentou tal ausência de sensibilidade.

A ausência de sensibilidade bacteriano à um determinado fármaco, culmina na anulação da utilização deste como medida terapêutica, contribuindo assim para o iniciar do quadro de sepse, que por sua vez, sujeitos acometidos por esta possuem custo estimado de internação avaliado em R\$ 3.692.421.00 (US\$ 1.649.138.40) relacionado à alta hospitalar, ao foco infeccioso pulmonar e à faixa etária até 59 anos (BARRETO; DELLAROZA; KERBAUY; GRION, 2016). Ademais, outro estudo realizado na mesma instituição identificou que a sepse é a principal causa mortis em idosos admitidos e internados no serviço de urgência e emergência (VASCONCELOS; CAMPOS; DE REZENDE, 2018). Este dado corrobora com o último estudo multicêntrico realizado em Unidades de Terapia Intensivas sediadas no Brasil, em que se observou uma alta mortalidade, ultrapassando 50\% nos sistemas público e privado de saúde. Este estudo se conclui com uma projeção estimada de 290 casos de sepse a cada 100.000 habitantes, rendendo cerca de 420.000 casos ao ano, dos quais 230.000 morrem na UTI (GOULART; BARROS; MONTEIRO, 2017). O Instituto Latino americano de Sepse, afirma ainda que a sepse supera o infarto do miocárdio e o câncer como principal causa de morte hospitalar tardia a (INSTITUTO LATINO AMERICANO, 2015). 


\section{CONCLUSÃO}

Concluímos que se faz necessário inclusão de projetos de extensão vinculados as atividades, pois possibilitou novas oportunidades de aprendizado no campo prático. Com isso, nossas observações apontam para adoção de estratégias que minimizem a incidência de infecções associadas à corrente sanguínea, com foco na educação continuada de profissionais de saúde, além da implementação de medidas de prevenção, detecção e tratamento da sepse.

Este relato de experiência contribui para a manutenção do conhecimento na área de controle de infecção hospitalar, pois relata sobre os microorganismos veiculados em um grande hospital da rede pública municipal, do Rio de Janeiro, sendo portanto, localizado na região em que se concentra a maior rede de saúde do país, bem como permitirá aos profissionais de saúde local: repensar, adequar, instalar e promover práticas de controle dos microorganismos com ausência de sensibilidade reportados aqui.

Por fim, observamos a importância no rastreio de bactérias gram positivas identificadas em hemocultura nesse referido hospital com grande porcentagem de ausência de sensibilidade aos antimicrobianos entre as bactérias gram negativas, um fato preocupante e alarmante. Sobretudo, certo de que a análise fenotípica é um indicador de resistência, sugerimos que esta seja precedida e seguida da análise genotípica, para investigação da veiculação dos genes de resistência bacterianos.

\section{AGRADECIMENTOS}

À chefia do Serviço de Análises Clínicas e ao Centro de Estudos Doutor João Pinho Filho do Hospital Municipal Souza Aguiar. 


\section{REFERÊNCIAS}

AGÊNCIA Nacional de Saúde Suplementar. Caderno de Informacão Da Saúde Suplementar: Beneficiários, Operadoras e Planos, 2015.

BARRETO, M. F. C.; DELlAROZA, M. S. G.; KERBAUY, G.; GRION CMC. Sepse em um hospital universitário : estudo prospectivo para análise de custo da hospitalização de pacientes. Rev Esc Enferm USP, v. 50, n. 2, p.302-308, 2016.

BRASIL. Ministério da Saúde. Documento de Referência Para o Programa Nacional de Segurança Do Paciente. Brasília: Ministério da Saúde/Fundação Oswaldo Cruz, editora, 2014. Disponível em: http://bvsms.saude.gov.br/bvs/publicacoes/documento_referencia_programa_nacional_seg uranca.pdf.

BUTAYE, P.; LAMMENS, C.; GOOSSENS, H.; DAS, A.J.; XAVIER B.B.; MALHOTRAKUMAR, S. Colistin resistance gene mcr-1 harboured on a multidrug resistant plasmid. Lancet Infect Dis., v. 16, n. 3, p.283-284, . 2016.

ERFANIMANESH S., HASHEMI A. Global Dissemination of the mcr-1 gene. Arch Pediatr Infect Dis., v. 4, n.3, p. 2014-2016, 2016.

GOULART, R.; BARROS, A.; MONTEIRO, K et al. The epidemiology of sepsis in Brazilian intensive care units (the Sepsis PREvalence Assessment Database, SPREAD): an observational study. Lancet Infect Dis., v. 17, n. 11, p.1180-1189, 2017.

HERNÁNDEZ-BOU, S.; TRENCHS SAINZ DE LA MAZA, V.; ESQUIVEL OJEDA, J.N.; GENÉ GIRALT, A.; LUACES CUBELLS, C. Factores predictores de contaminación ante un hemocultivo con crecimiento bacteriano en Urgencias. An Pediatría, v. 82, n. 6, p. 426-432, 2015 .

INSTITUTO BRASILEIRO de Geografia e Estatística. Pesquisa Nacional de Saúde: 2013: Acesso e Utilização Dos Serviços de Saúde, Acidentes e Violências : Brasil, Grandes Regiões, Unidades Da Federação, Municípios Das Capitais e Distrito Federal. Rio de Janeiro. 2015. Disponível em: ftp://ftp.ibge.gov.br/PNS/2013/pns2013.pdf

INSITUTO LATINO Americano para Estudos da Sepse. Sepse: Um Problema de Saúde Pública. Brasília: Conselho federal de Medicina, 2015. https://ilas.org.br/assets/arquivos/upload/Livro-ILAS(Sepse-CFM-ILAS).pdf\%0A. 
LONG, B.; KOYFMAN, A. Best Clinical Practice: Blood Culture Utility in the Emergency Department. J Emerg Med., v. 51, n. 5, p. 529-539, 2016.

SINGER, M.; DEUTSCHMAN, C.S.; SEYMOUR, C et al. The third international consensus definitions for sepsis and septic shock (sepsis-3). JAMA - J Am Med Assoc., v. 315, n. 8, p.801-810, 2016.

VAN WALRAVEN, C.; WONG, J. Independent influence of negative blood cultures and bloodstream infections on in-hospital mortality. BMC Infect Dis., v. 14, n. 1, p.1-11, 2014.

VASCONCELOS C. B.; CAMPOS G. C.; DE REZENDE B. G. T. Perfil de causa mortis em idosos internados em um serviço público de urgência e emergência: evidências clínicas. Acad Rev Científica da Saúde, v. 3, n. 2, p. 29-35, 2018.

WORLD HEALTH Orgaznization. Global Priority list of antibiotic-resistant bacteria to guide research, discovery and development of new antibiotics. April 2017. Disponivel em: $\quad$ https://www.who.int/medicines/publications/WHO-PPLShort_Summary_25FebET_NM_WHO.pdf. 